$$
\text { DOE/ER/4O2 } 71-T 2
$$

DOE/ER/40271--T2

PROGRESS REPORT

DE92 006532

\title{
Central Collisions of Heavy Ions
}

October 1, 1990 to September 31, 1991

\section{DISCLAIMER}

This report was prepared as an account of work sponsored by an agency of the United States Government. Neither the United States Government nor any agency thereof, nor any of their emplnyees, makes any warranty, express or implied, or assumes any legal liability or responsibility for the accuracy, completeness, or usefulness of any information, apparatus, product, or process disclosed, or represents that its use would not infringe privately owned rights. Reference herein to any specific commercial product, process, or service by trade name, trademark, manufacturer, or otherwise does not necessarily constitute or imply its endorsement, recommendation, or favoring by the United States Government or any agency thereof. The views and opinions of authors expressed herein do not necessarily state or reflect those of the United States Government or any agency thereof.

\author{
prepared by \\ Sun-yiu Fung \\ Principal Investigator \\ DOE Nuclear Science Program \\ Grant Number DE-FG03-86ER40271
}

October 1991 


\section{CONTENTS}

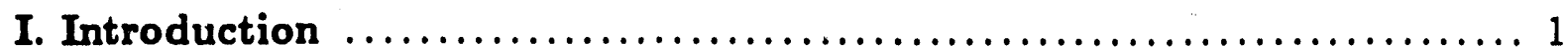

II. Heavy-ion Collisions Study at AGS energies $\ldots \ldots \ldots \ldots \ldots \ldots \ldots \ldots \ldots$

A. E802 Particle Production $\ldots \ldots \ldots \ldots \ldots \ldots \ldots \ldots \ldots \ldots \ldots \ldots \ldots \ldots$

(1) Intermittency analysis for E802 $\ldots \ldots \ldots \ldots \ldots \ldots \ldots \ldots \ldots \ldots \ldots \ldots$

(2) Comparison of $\mathrm{p}+\mathrm{A}$ and $\mathrm{Si}+\mathrm{Au}$ Collisions $\ldots \ldots \ldots \ldots \ldots \ldots \ldots \ldots \ldots \ldots \ldots \ldots \ldots \ldots \ldots$

(3) Anti-proton production for $\mathrm{Si}+\mathrm{A}$ Collisions $\ldots \ldots \ldots \ldots \ldots \ldots \ldots \ldots \ldots 2$

(4) $\pi$-correlation in $\mathrm{Si}+\mathrm{Al}$ and $\mathrm{Si}+\mathrm{Au}$ collisions $\ldots \ldots \ldots \ldots \ldots \ldots \ldots \ldots$

B. E859 Particle Production .................................. 3

(1) Second Level Particle Identification Trigger $\ldots \ldots \ldots \ldots \ldots \ldots \ldots \ldots \ldots . \ldots$

(2) E859 Runtime Equipment Status Monitor ..................... 3

(3) E859 Pass12 Data Preprocessing Analysis ..................... 4

(4) HBT Analysis $\ldots \ldots \ldots \ldots \ldots \ldots \ldots \ldots \ldots \ldots \ldots \ldots \ldots \ldots \ldots \ldots, 4$

C. E866 Gold Beam Experiment $\ldots \ldots \ldots \ldots \ldots \ldots \ldots \ldots \ldots \ldots \ldots \ldots$

D. RHIC Proposals and R\&D f........................... 5

III. Study of particle production at Bevalac energy $\ldots \ldots \ldots \ldots \ldots \ldots \ldots 6$

A. Pair Correlation Study with HISS $\ldots \ldots \ldots \ldots \ldots \ldots \ldots \ldots \ldots \ldots$

B. Collective Flow Study with Streamer Chamber $\ldots \ldots \ldots \ldots \ldots \ldots 7$

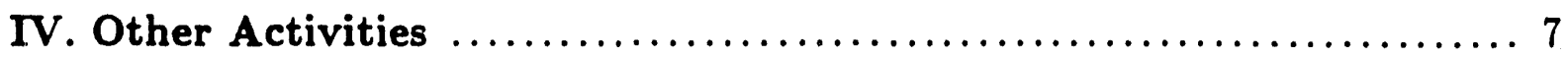

A. BNL Neutrino Oscillation experiment $\ldots \ldots \ldots \ldots \ldots \ldots \ldots \ldots \ldots 7$

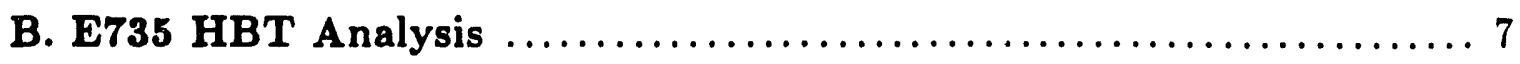

C. Double beta decay experiment at Idaho $\ldots \ldots \ldots \ldots \ldots \ldots \ldots \ldots, 7$

V. Summary and Outlook $\ldots \ldots \ldots \ldots \ldots \ldots \ldots \ldots \ldots \ldots \ldots \ldots \ldots \ldots, \ldots$ 


\section{Introduction}

This report describes the activities of the Heavy Ion Physics Group at the University of California, Riverside from October 1, 1990 to September 30, 1991. During this period, our program focuses on particle production at AGS energies, and correlation studies at the Bevalac in nucleus nucleus central collisions. We participated in the preparation of letters of intent for two RHIC experiments - the OASIS proposal and the Di-Muon proposal - and worked on two RHIC R\&D efforts - a silicon strip detector project and a muon-identifier project. A small fraction of time was also devoted to physics programs outside the realm of heavy ion reactions by several individuals.

\section{Heavy-ion Collisions Study at AGS energies}

We have started on the second phase of the particle production study with heavy ion collisions at the AGS. The E802 collaboration completed its final run in 1989, and is continuing works on several data analyses, including the intermittency study carried out at Riverside. In this period, the $\mathrm{E} 859$ has its first long production run, collecting data for particle correlation study, strange particle production, and high momentum transfer phenomenon, with the full implementation of a second level particle identification trigger. We are also planning a silicon strip detector for the approved E866 gold beam experiment.

\section{A. E802 Particle Production}

The Riverside Group has been a part of the BNL E802 collaboration since 1986. This is the first major experiment utilizing the $14.5 \mathrm{GeV} / \mathrm{c}$ heavy ion beam at the AGS. Its goal is to study particle production in nucleus nucleus central collisions. The apparatus consists of a large aperture magnetic spectrometer, with segmented Cherenkov counters, scintillation counter arrays, time projection chamber and wire chamber for tracking and particle identification; a smaller aperture gas Cherenkov system tuned to identify higher momentum particles; a lead glass array to measure total neutral energy; a streamer tube array to determine total multiplicity and measure pseudorapidity of emerging particles; and a small total absorption sampling calorimeter to measure the total kinetic energy of the projectile fragments at small forward angles. The data collection was completed in 1989, and investigations on many aspects of partic'e production are being carried out.

(1) Intermittency analysis for E802

A large concentration of particles in narrcw rapidity regions was observed in ultra high energy cosmic ray experiment such as JACEE. The concept of intermittency 
was applied to this data analysis to understand the underlying physics of multiparticle production. Subsequently, this phenomenon was studied in electron-positron collisions, hadron-hadron, hadron-nucleus, and nucleus-nucleus collisions. However, the interpretation and the cause of intermittency is far from clear. In the Brookhaven experiment E-802, Target Multiplicity Array (TMA) can measure particle pseudorapidity from -1.2 to 3.1 with almost full azimuthal coverage. An intermittency analysis was carried out on these data to study short range multiparticle correlations. Factorial moments for the central collisions between ${ }^{16} \mathrm{O}$ projectiles with $14.6 \mathrm{~A} \cdot \mathrm{GeV} / \mathrm{c}$ and targets of $\mathrm{Al}, \mathrm{Cu}$ and $A g$ have been calculated with bin size up to $\delta \eta=0.06$. An intermittency behaviour, power-law growth of moments with decreasing bin size, was seen up to $\delta \eta \sim 0.1$, with stronger effect in lighter target than heavier target. These preliminary results have been submitted by J. Kang to the Quark Matter ' 91 Conference to be held in Gatlinburg, Tennessee in November. (Appendix A)

\section{(2) Comparison of $\mathrm{p}+\mathrm{A}$ and $\mathrm{Si}+\mathrm{Au}$ Collisions}

The considerably enhanced yield of $\mathrm{K}^{+} / \pi^{+}$in central Si+Au collisions, in comparison with that in $\mathrm{p}+\mathrm{p}$ collisions, is very intriguing. To study this phenomenon in greater details, data were collected and analyzed for $\mathrm{p}+\mathrm{Be}, \mathrm{p}+\mathrm{Au}$, and $\mathrm{Si}+\mathrm{Au}$ collisions.

The invariant cross sections with respect to the transverse kinetic energy are all exponential in form. The inverse slopes are similar for proton, kaons and pions in $\mathrm{p}+\mathrm{A}$ reactions, $\sim 140-160 \mathrm{MeV}$. For $\mathrm{Si}+\mathrm{Au}$, pions have similar slopes, but the protons and the $\mathrm{K}^{+}$have larger values, $\sim 200-220 \mathrm{MeV}$ at $1.2 \leq \mathrm{y} \leq 1.4$. The particle yields as function of rapidity are extracted with the assumption of a single exponential function for each of the invariant cross section distribution. Within the measured rapidity, the ratio of the integrated yield for $\mathrm{Au}$ and Be targets is obtained: $1.08 \pm 0.03$ for $\pi^{+}, 1.81 \pm 0.18$ for $\mathrm{K}^{+}$and $1.95 \pm 0.02$ for proton. Thus, from Be to $\mathrm{Au}$, the $\pi^{+}$yield remains practically constant while the $\mathrm{K}^{+}$yield rises as strongly as protons. The $\mathrm{K}^{+}$yield per nucleon for $\mathrm{Si}+\mathrm{Au}$ is even higher. For $\mathrm{p}+\mathrm{Be}$, the spectra are rather broad. For gold targets, the peaks shift towards and below the participant rapidity. This investigation will be expanded to cover a larger rapidity range in E859.

These results were reported at the QM'90 and published in the Physical Review Letters. (Appendix B and C) The features associated with $\mathrm{K}^{+}$production - the large slope parameter, the spectrum shape and magnitude - should present a challenge and powerful constraint to any proposed theory for nucleus nucleus collisions at AGS energies.

\section{(3) Anti-proton production for Si+A Collisions}

The invariant cross section of anti-proton was measured in $\mathrm{Si}+\mathrm{A}$ collisions, and fitted to an exponential in transverse mass. The inverse slope parameter was found to be the smallest in comparison with pion, kaon and proton, $\sim 140 \mathrm{MeV}$, and similar to that in $\mathrm{pp}$ and $\mathrm{pA}$ collisions. This result is not consistent with the hydrodynamic model, which predicts a similar slope parameter for anti-proton and proton. The ratios of antiproton to pion and kaon decrease with increasing target mass. The cause could be due to the large anti-proton annihilation cross section, the degradation of projectile energies 
while traversing the medium. This study is limited by statistics ; the total sample of anti-proton is $\sim 1,000$. We are collecting a much larger sample in E859 for further investigation. These results were reported at the QM'90 (Appendix D), and a full letter has been accepted for publication in Physics Letters.

(4) $\pi$-correlation in $\mathrm{Si}+\mathrm{Al}$ and $\mathrm{Si}+\mathrm{Au}$ collisions

Hanbury-Brown-Twiss (HBT) interferometry technique was applied to the pion pairs of identical charges, both positive and both negative, to extract the interaction source size. The transverse size is comparable to the projectile radius, $4.7 \mathrm{fm}$, for both targets. The "chaoticity" parameter, $\lambda$, is greater than 0.5 . These preliminary results were reported at the QM'90. (Appendix E)

\section{B. E859 Particle Production}

E859H collaboration extends the investigation of particle production started by E802 towards rarer events associated with high transverse momentum, interactions with small cross sections such as strange particle and anti-proton production and multi-particle correlation. The major addition to the E802 apparatus is a second level on-line programmable trigger for particle identification. The UCR group has been active primarily in the following areas:

\section{(1) Second Level Particle Identification Trigger}

For the second E859 run, a second level trigger associated with the magnetic spectrometer is implemented to select specific event topologies. The input from the wire chambers as well as time of flight modules was processed for each track. The resulting data were fed into pre-calculated look-up tables, stored in five sets of Memory Look-up Units (MLU), to extract the momentum and the mass of this track. Information from all tracks emerging from the magnetic spectrometer was combined to from, for example, $\mathrm{K}^{+}, \mathrm{K}^{-}$/anti-proton, $2 \pi, 2 K^{-}, \mathrm{K}^{+} K^{-}$triggers. The operation of this trigger is programmable at run time. J. Kang is a member of the Second Level trigger group. He carried out the simulation of particle trajectories through the spectrometer system for hit locations in wire chambers and time of flight modules, developed the code to generate the look-up tables, and maintained a set of particle identification tables. The run was very successful. For example, 5.3 million triggers were recorded for the $2 \mathrm{~K}^{+}$ trigger run, and close to 20,000 good $K^{+}$pairs is expected from the 1991 Spring data set.

(2) E859 Runtime Equipment Status Monitor

The E859 program FSCALE was resurrected by P. Beery for the spring 1991 heavy ion run at BNL. The new version of the program underwent major revision to enhance its performance as a real-time monitor of the data acquisition hardware. It provided 
capability for remote observation of the current and past run status. This allowed "Experts" including those not at the counting house to determine when a failure occurred and to commence remedial actions much sooner. Beery, with the help of James Chang and $R$. Seto adapted and largely rewrote some code which monitors beam and trigger rates on-line from scalers coming from the AGS and the detector electronics. FSCALE was fully operational as intended in the 1991 run.

The current plans for FSCALE are to fully implement the histogram feature which was added on a basic level for the last run and to improve the user interface. Fault checking/warning routines will also be added.

\section{(3) E859 Pass12 Data Preprocessing Analysis}

Riverside is involved with the processing of the data taken sofar in the two runs of E859. We are running the reconstruction routines (Pass12). This pass augments the raw data chamber hits with tracking and momentum information. The data is then ready for use for subsequent physics analysis. The collaboration plans to have this reconstruction, and analysis done in time to present results at the Quark Matter ' 91 Conference. The majority of the work in setting up the reconstruction at UCR has been done by Beery. The work in running this large amount of data has been shared between Beery and Seto. Dividing the load equally between MIT, Columbia and UCR, each institution is processing $1 / 3$ of the data which consumed about 1000 hours of VAX CPU time. For this purpose the UCR heavy ion group has committed 3 Gbytes of disk space and several VAX $31 / 76$ Workstations. Our participation in this effort is possible because of the large amount of disk space and CPU power purchased recently using the campus fund granted to $R$. Seto as an initial complement. This money is due to expire at the end of this academic year.

The data processing set-up would give Riverside the capabilities to handle the increase in data expected with the upcoming Au-Au E866H BNL runs.

\section{(4) HBT Analysis}

We plan to look at two topics in the E859 Si+A data. P. Beery will be working on the $2 \pi \mathrm{HBT}$ data analysis as a function of rapidity. UCR will concentrate on the source shape analysis by examining data taken with the spectrometer located at angles $14^{\circ}$, $24^{\circ}, 36^{\circ}$ relative to the beam line. This work has just been started.

R. Seto plans to investigate something more speculative. For the last few months he has looked at the $\mathrm{K}^{+} \mathrm{K}^{-}$mass distribution in the mass region of the phi meson, to see if there in an enhancement in its production cross section as seen by NA38 at CERN. This may be possible but it will depend critically on the efficiency of the second level trigger at 5 degree spectrometer setting.

\section{E866 Gold Beam Experiment}

The booster project at BNL will make available for the first time, very heavy projec- 
tiles (gold) for heavy ion experiments. E866 will use the E802/E859 detector, upgraded to handle higher multiplicities. It will add a brand new small aperture forward spectrometer. The Target Multiplicity Array (TMA) is expected to be hit by 600 particles in the froward wall; the existing streamer tube granularity is too coarse for this environment. The UCR group is proposing to build a Silicon Microstrip TMA. The granularity provided by silicon strip detectors makes the high multiplicities of gold-gold collisions measurable. Occupancy rates of $10 \%$ for each microstrip are possible for a reasonable cost. Electronic costs per channel are small because of the availability of integrated chips incorporating large sumbers of channels, typically 100 per chip. Many of these chips include a zero-suppression feature which greatly helps in reducing the readout time and the amount of data collected from the device. The initial proposal for this detector has been primarily the work of Seto and Beery. Monte-Carlo calculations have been done to select the best geometry and to look at questions of rapidity coverage, and the response of the detector to various particle types. Further work to be done in the near future will include the study of delta ray contamination, possible background caused by multiple scattering of particles into the main spectrometer, and the timing for electronic read-out.

Much of the purchasing of equipment has been done in the last few months. Since very little was available at $\mathrm{UCR}$ in the way of oscilloscopes and fast electronics, this all had to be purchased using the initial complement money of $R$. Seto. Further purchases of phototubes, power supplies and additional electronic equipment are still necessary. A first sample detector has just arrived at UCR from SI, a Norwegian company. Within the next two months the design of the first serious prototype will be finished and we will be soliciting bids from various compauies. In the next two months the collaboration will be deciding whether or not to officially pursue this project. If the response is positive we anticipate help from a group from BNL and the electronics group at Nevis. We have already received assistance from the Silicon Detector group here at UCR which is involved in SSC development work, as well as the construction of a silicon vertex detector at D0. This work also serves to incorporate us into the R\&D work for RHIC. R. Seto had just joined the Silicon effort for the OASIS collaboration (Appendix F), before the merging of the various RHIC collaborations into one unified effort. We hope to become part of the Silicon effort in that new collaboration, RE2, when it is formed. Again, It must be emphasized that this work was possible only because of the campus funds given to $\mathrm{R}$. Seto as an initial complement. In order to continue this work, money must be allocated to the group at UCR for permanent equipment in the future by DOE.

\section{RHIC Proposals and R\&D}

Riverside has been actively involved in two letters of intent for future RICH experiments: the OASIS proposal and the di-muon proposal. The former emphasized a multi-signal approach where many physics parameters will be measured as function of transverse energy density in different rapidity intervals. The latter concentrated on the $J / \psi$ production. 
$J / \psi$ suppression was observed at CERN energies in nucleus nucleus collisions. However, the results could be explained by many conventional mechanisms other than the formation of quark-gluon-plasma. To resolve this question, it is necessary to measure its production as a function of momentum transfers. A large muon detector to cover a wide range of transverse momentum was designed for RHIC in the Di-muon proposal. At UCR tracking simulation codes for the spectrometer of the Dimuon Experiment at RHIC has been developed by J. Kang using GEANT. Geometries of the toroid magnet and drift chambers in the barrel and endcaps were implemented. Tracks have been simulated using the magnetic fields in the proposal to study the behaviour of lower momentum particles as well as the amount of the vertical focusing (bending in the azimuthal angle). This tracking simulation codes will be essential when full scale track reconstruction software is to be developed.

R. Seto has been involved with the OASIS proposal for RHIC. He was on the institutional board of the group. His main contribution has been to study the possibility of measuring di-electrons with the OASIS detector, which was done together with an undergraduate student at UCR, Michael Towne. Di-electrons are interesting for several reasons in the search for the QGP. First, a measurement of the production of the vector mesons which decay to $\mathrm{e}^{+} \mathrm{e}^{-}$and their relative enhancement as a function of centrality, $p_{t}$, or beam particle type might be an indication of screening effects or of strange quark concentration inside the plasma. Secondly, any variation in the mass, widths, and branching ratios might be an indication of a chiral symmetry restoration. Finally, the di-electron continuum, would be a measure of the virtual direct photon yield, which can be related to the temperature of the plasma. These calculations were among the driving forces in the design of the OASIS detector.

At the September BNL PAC meeting, both the OASIS and also the Di-muon proposal were not approved. Collaborators were asked to join a new effort instead, RHIC Experiment 2 (RE2). The physics emphasis for the first stage was on the photon/lepton production study. The UCR group is taking an active role in the new collaboration. We also plan to join the effort to design and build a silicon multiplicity detector. R. Seto is now a member of the Conceptual Design Group (ĆDG) for RE2.

\section{Study of particle production at Bevalac energy}

\section{A. Pair Correlation Study with HISS}

Riverside is undertaking the proton proton correlation analysis using the data of $\mathrm{La}+\mathrm{La}$ collisions collected with the HISS at the Bevalac. As we had already studied the $\pi^{-}$correlations, this would give a unique set of data where both pion and proton interferometry is studied under the same physics and experimental conditions. James Chang has calibrated the time-of-flight system and separated the proton from the pion. G.H. Xu is working on the theoretical predictions. Some preliminary results will be presented at the Interferometry Workshop held at MSU before the annual Division of Nuclear Physics Meeting. Another talk of the HISS collaboration will be presented by W. Christie of LBL on pion correlation in La+La collisions. 
Results on pion correlations in $\mathrm{Ar}+\mathrm{KCl}, \mathrm{Ar}+\mathrm{La}$, and $\mathrm{Xe}+\mathrm{La}$ collisions at Bevalac energies were reported at the QM'90 (Appendix G). The rms source sizes are close to the radius of the projectile.

\section{B. Collective Flow Study with Streamer Chamber}

To study the properties of the equation of state (EOS) of compressed nuclear matter, many observables such as pion production, kaon production, collective sideward deflection of the reaction product in terms of flow angles, transverse momenta have been proposed. In the past, we have analysed our streamer chamber data with the transverse flow analysis proposed by Danielewicz and Odyniec. However, the accuracy of this method depends heavily on the definite particle identification, and the small dispersion of the reaction plane which is affected by track finding efficiency.

To overcome these difficulties, an azimuthal correlation function analysis is adopted. We show that this correlation is sensitive to the parameters of the equation of state. These results were published in Phys. Rev. C44 (Appendix H). Similar analysis could be applicable to data collected at AGS energies.

\section{Other Activities}

\section{A. BNL Neutrino Oscillation experiment}

R. Seto has also spent sorne time working on the final stages of data analysis and paper writing for E776, a neutrino oscillation experiment at BNL. Together with a graduate student at Columbia, new limits were set on muon neutrino oscillations. This work is now submitted to PRL, and was recently presented at the Washington meeting of the APS and at the Intersections Conference in Tucson. A paper on the Flash ADC system has been completed and published in the past year. Several other papers are in progress.

\section{B. E735 HBT Analysis}

Over the last year work has continued on the HBT analysis of the Fermilab E735 experiment's final data sample. The source size and shape have been examined as a function of charged multiplicity per unit of pseudorapidity $\frac{d N_{c}}{d \eta}$ and transverse momentum $P_{T}$ of the pion pair. This data was taken with the Fermilab $P \bar{P}$ collider with center of mass energy $\sqrt{s}=1.8 \mathrm{TeV}$. Future plans include the completion of this analysis and direct comparison with the E859 results. Every effort will be made to make the analysis of the E859 and E735 data samples substantially identical in order to facilitate this comparison.

\section{Double beta decay experiment at Idaho}

This experiment had been building more detectors to increase the number of its 
lithium-drifted silicon detectors four times over the previous experiment for last few years and developed new technique to make cleaner molybdenum sources. Better sensitivities in the measurements of the half-life time of molybdenum beta decay with two neutrinos and no-neutrino will be possible by taking data with the expanded detectors and the cleaner sources. New data taking started this spring and I spent few weeks at Idaho early this summer to help data taking and plan to make another trip near the end of this year for the same purpose.

\section{Summary and Outlook}

We have applied the intermittency analysis to the E802 oxygen data. The preliminary results proved this feasible, and we shall continue to investigate in depth this phenomenon at AGS energies.

The data processing system for the reconstruction of tracks has been established at Riverside. A third of the E859 data was processed through locally. This would release the bottleneck in data processing in the future. We will refine the software components of the Second Level Particle Identification Trigger, and the remote monitoring package, FSCALE, for the 1992 Spring run. We will take on the $2 \pi$ interferometry analysis, as well as the study of $K^{+} K^{-}$resonance formation.

For the forthcoming E866 experiment, we will investigate the feasibility and the practicality of implementing a silicon microstrip detector to replace the streamer tube TMA wall. This effort is complementary to the silicon strip detector $R \& D$ efforts for RHIC.

We will continue our Bevalac experimental analyses on both the HISS and the streamer chamber data. We shall have a very productive year. 

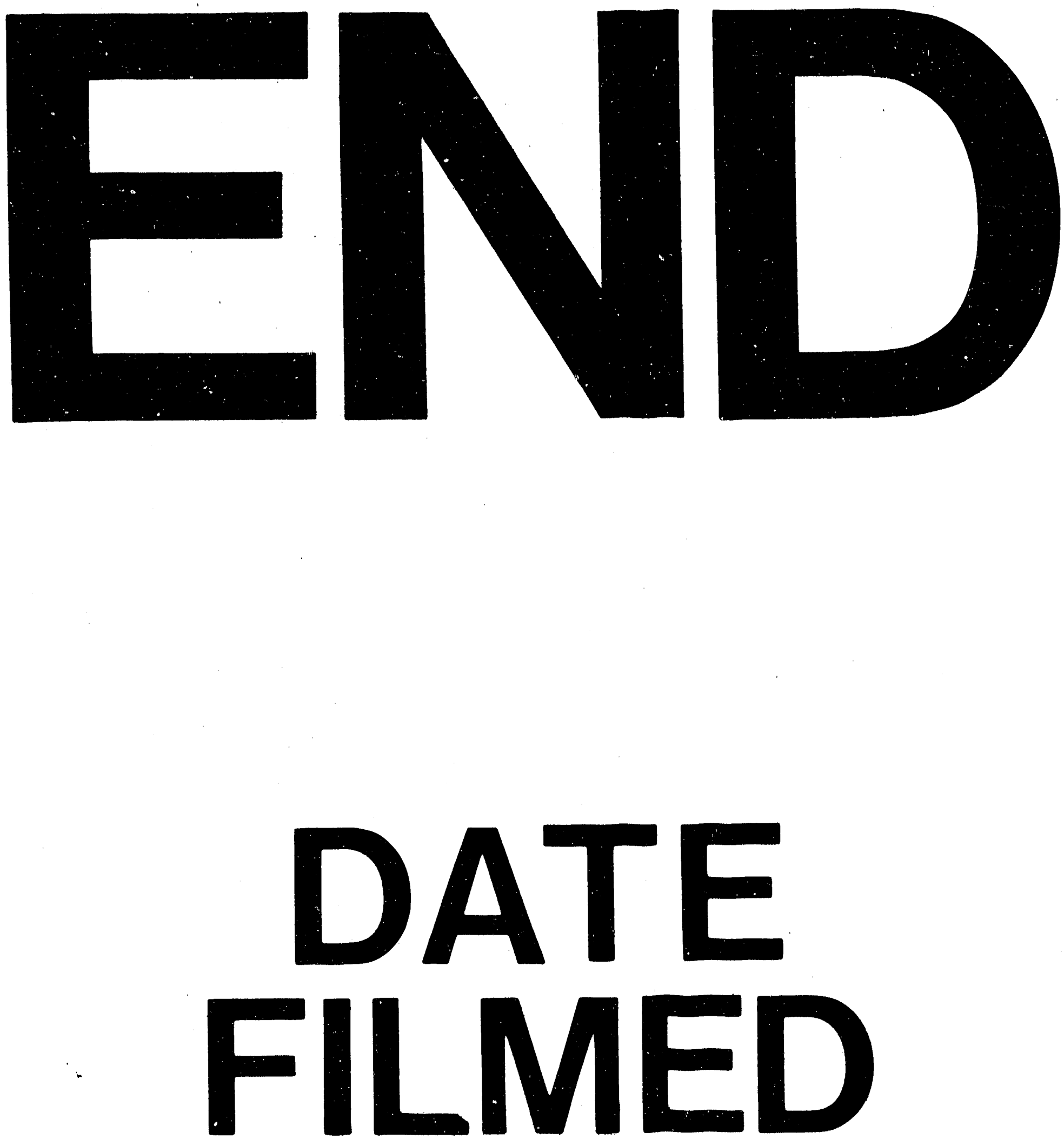

1

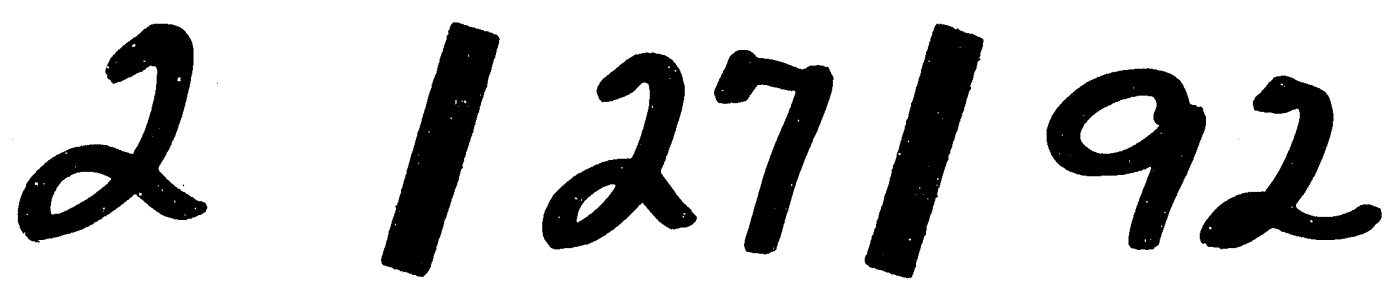


